\title{
Acquisition of images and parameter calculations for the automatic identification of molecules in a thin-film extract combined with laser
}

\author{
Théodore Guié Toa Bi ${ }^{1}$, Sié Ouattara ${ }^{1, *}$, Jérôme Nango Alico ${ }^{1}$, Alain Clement ${ }^{2}$ \\ ${ }^{1}$ Laboratory of Signals and Electrical Systems (L2SE), National Polytechnic Institute Houphouët Boigny, BP 1093 \\ Yamoussoukro, Côte d'Ivoire. \\ ${ }^{2}$ Institute of Technology of Angers (IUT) / University of Angers, France. \\ *Correspondence: sie_ouat@yahoo.fr; Tel.: +225-57 127595
}

\begin{abstract}
This paper presents the automation of the thin-layer chromatography technique whose separation and identification of molecules present in a mixture are currently done manually and laboriously [1]. We have therefore found an interest in automating this technique. In this part, the method implemented comprises 2 steps. First we proceeded to the segmentation of the spots obtained on the chromatographic plate. We then developed a program to identify families of molecules such as coumarins, terpenes, tannins, flavonoids, polyphenols, etc. by calculating segmentation parameters such as standard deviation, entropy, average pixel intensity from an algorithm on the matlab software. Finally our results have been compared to the results obtained by the traditional identification technique in laboratories. Some similarity between the two results obtained shows the reliability and the robustness of our technique.
\end{abstract}

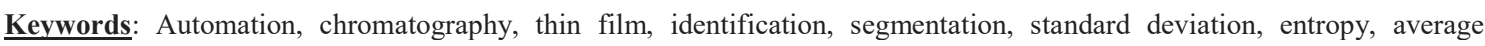
intensity, algorithm, matlab.

\section{Introduction}

Traditional medicine based on medicinal plants, survives and tends to develop in parallel with modern medicine in developing countries. According to the World Health Organization (WHO) in 2014, nearly 80\% of people in developing countries use traditional medicine for primary health care; hence the need for scientific valorization of medicinal plants [2]. A majority of the world's population, especially in developing countries, is treated only with traditional herbal remedies [3]. Each plant can contain several thousand different compounds whose various uses are aimed at overcoming suffering and improving the health of men. [4]

In addition, the identification of families of molecules in the leaves of plants (extracts) are of great interest in laboratories of natural substances. In these laboratories all research is based on plants (stem, leaves, roots, seeds) and mostly works in the context of the revaluation of traditional medicine based on plants. [5]

However, the separation and identification of molecules is done manually, always referring to the data in the literature, which is slow and very laborious. We have therefore found an interest in automating the identification technique based on algorithms run on Matlab software version 2016. [6]

For this work, we used three (03) plants that are: Alchornea cordifolia, Paullinia pinnata (Sapindaceae), Trema guineensis (Ulmaceae).

We have first described the material and methods in which we present the segmentation of the digital color images of the extracts acquired on the plate as well as the device of experimentation acquisition of these images. The second step is a lowlevel image processing of segmentation parameter calculations such as entropy, standard deviation, and average pixel intensity. Finally, we compare our results with the results obtained by the traditional identification technique.

\section{Materials and methods}

2.1 Material

\subsubsection{Chemical reagents and equipment}

All the chemicals used are of analytical quality. The solvents and reagents employed were purchased commercially from Polychimie (Ivory Coast). For thin-layer chromatography, we used silica gel 60 F254 chromatoplates on an aluminum support. The different devices used for our analyzes are as follows:

- A RETSCH brand electric grinder, type SM 100

- A brand balance Sartorius (Germany)

- Burchi RII Rotary Evaporator

- A magnetic stirrer

- A UV-Visible spectrophotometer

- A camera

$\checkmark \quad$ Spatial resolution: 2448x3264 (8 M pixels)

$\checkmark$ Tonal resolution: 24 bits (color)

$\checkmark$ Color space: RGB 
$\checkmark \quad$ Camera model: Infinix

$\checkmark \quad$ Focal length: F/2

$\checkmark$ Exposure time: $1 / 13$ seconds

$\checkmark \quad$ ISO sensitivity: ISO-864

$\checkmark \quad$ Focal distance: $4 \mathrm{~mm}$

$\checkmark \quad$ Picture format: JPG image

- Glassware (round bottom flask, beakers, etc.)

- UV lamp (366 nm, $254 \mathrm{~nm})$

\subsubsection{Plant material}

Plant organs consisting mainly of leaves were all harvested from the forest relic of Nangui Abrogoua University in Côte d'Ivoire (see Figure 1). The plant species have been identified according to the herbaria available at the National Center for Floristics $(\mathrm{CNF})$.

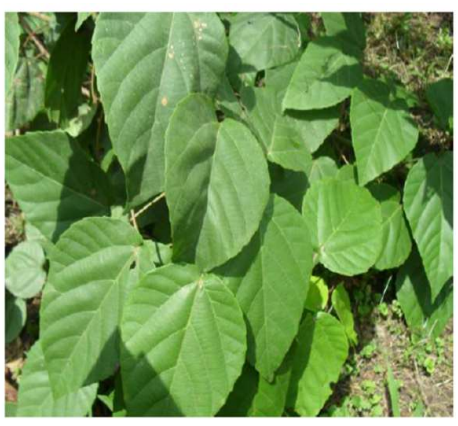

(a)

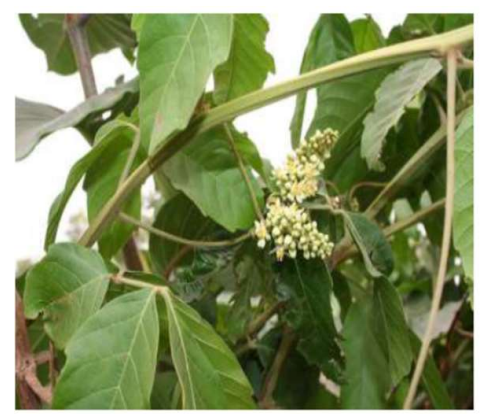

(b)

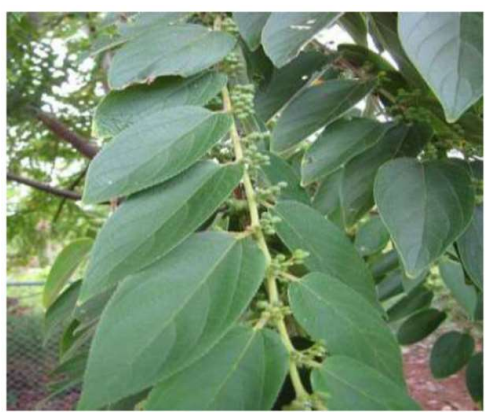

(c)

Figure 1. (a): Leaves of Alchornea cordifolia, (b): Leaves of paullinia pinnata, (c): Leaves of Trema guineensis

\subsection{Methods}

\subsubsection{Selective extraction of some secondary metabolites}

The hydromethanolic extracts of the various leaves were treated respectively with $3 \times 20 \mathrm{ml}$ of hexane, chloroform, ethyl acetate and n-butanol (see figure 2). The various organic fractions were concentrated under reduced pressure on a rotary evaporator and then stored in a refrigerator. Thus, for each crude extract, we obtain selective extracts distributed as follows: hexanic, chloroformic, ethyl acetate which were used for phytochemical screening.

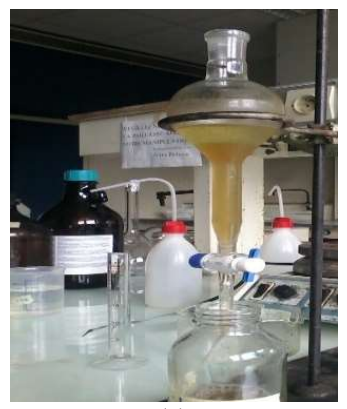

(a)

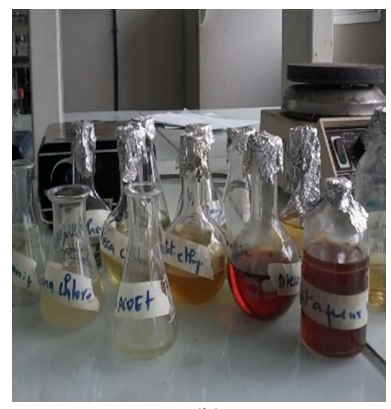

(b)

Figure 2.(a) Fractionation process of the hydromethanic extract and (b) Hexanic fraction, chloroform, ethyl acetate

\subsubsection{Photochemical screening and image acquisition device}

The photochemical screening was carried out according to the analytical procedures described in the work of [7], [8] and [9]. Drops of each selective extract are deposited using a capillary on the points $(01 \mathrm{~cm}$ from each other) of the baseline drawn $1 \mathrm{~cm}$ from the bottom of the chromatoplates (silica gel $60 \mathrm{~F} 254$ ). The deposits are allowed to dry for a moment before introducing the plates into the chromatograph vessel containing the migration solvent. After migration and drying with a hair dryer, the chrotograms are revealed with reagents specific to the phytochemistry groups sought, then visualized first in the visible and then under a UV lamp at $366 \mathrm{~nm}$ (see Figure 3). The different developer systems used are:

- Hexane / AcOEt (20: 4, v/v) for hexanic fractions.

- $\mathrm{CHCl} 3 / \mathrm{AcOEt} /$ hexane (10: 10: 5, v/v/v) for the chloroform fractions.

- AcOEt / CH3OH / H2O / CHCl3 (18: 2,4: 2,1: 6, v/v / v / v) for the ethyl acetate fractions. 


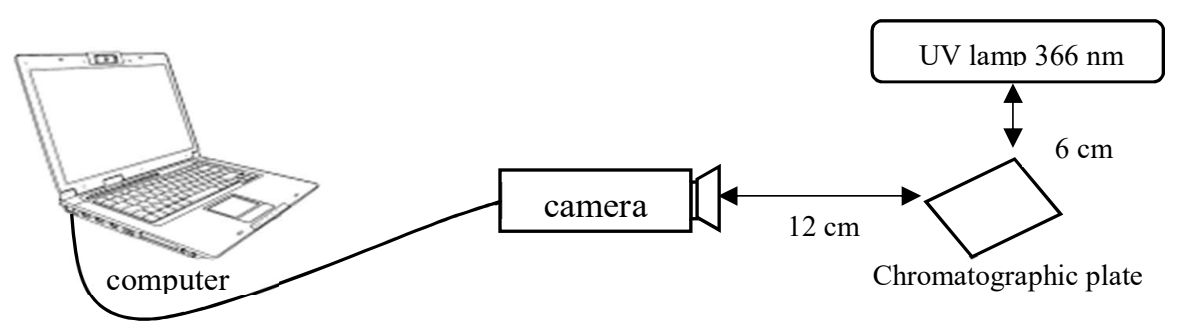

Figure 3. Image acquisition device on the chromatographic plate

\section{Results and discussions}

\subsection{Acquisition of images on the chromatographic plate}

For each extract, we obtained images on the chromatographic plates. Figure 4 shows some of the images obtained.

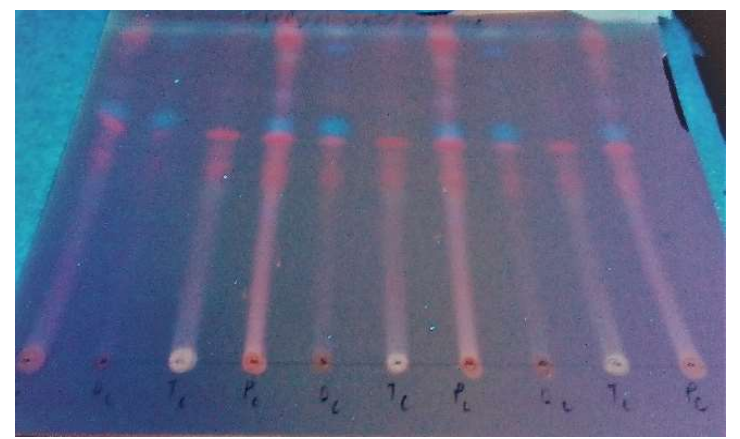

Figure 4a.UV chromatogram at $366 \mathrm{~nm}$ chloroformic extracts

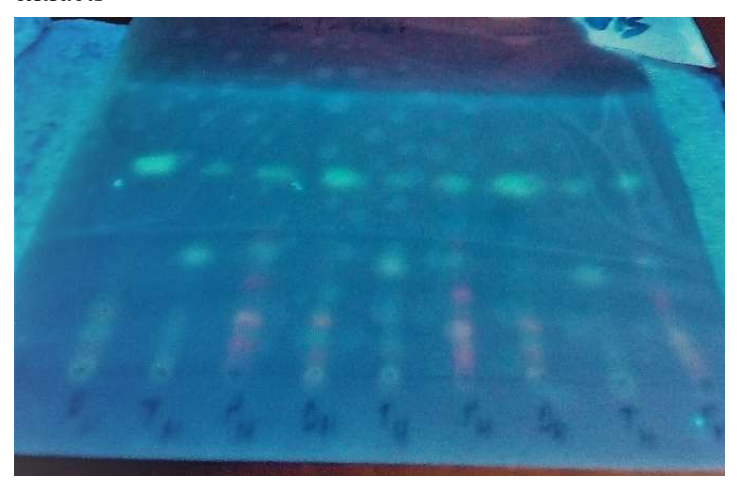

Figure 4c. UV chromatogram at $366 \mathrm{~nm}$ of hexane extracts after treatment with Libermann-Büchard

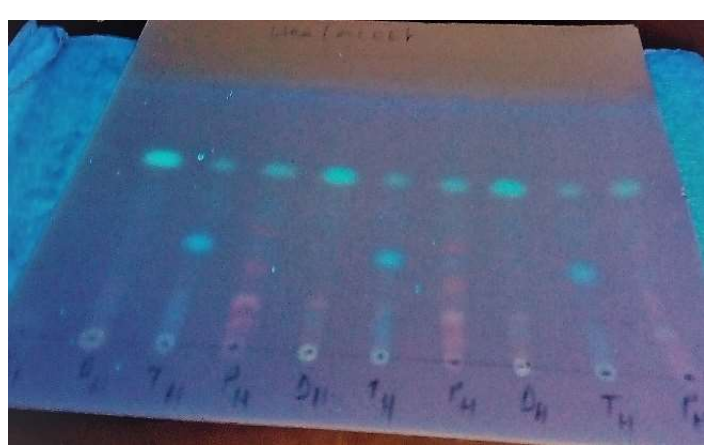

Figure 4b. UV chromatogram at $366 \mathrm{~nm}$ of hexane extracts

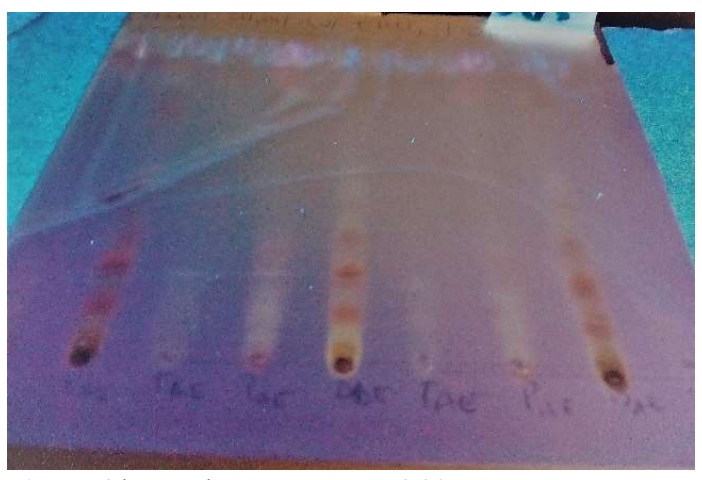

Figure 4d. UV chromatogram at $366 \mathrm{~nm}$

Ethyl acetate extracts after treatment with $\mathrm{KOH}$ 


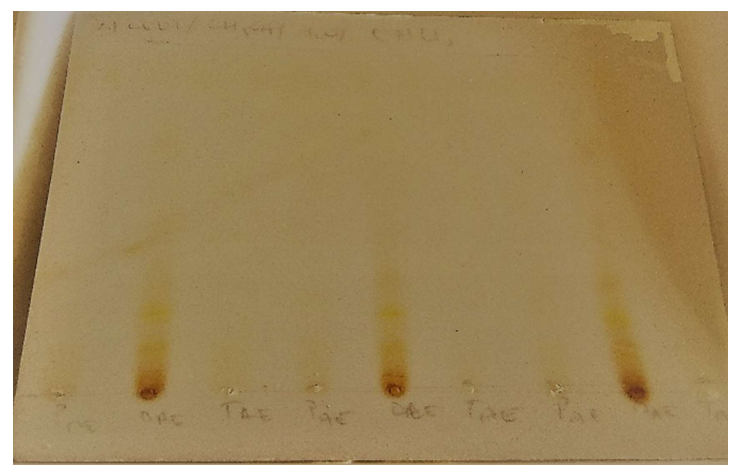

Figure 4e. Chromatogram in the visible ethyl acetate extracts after treatment with $\mathrm{KOH}$

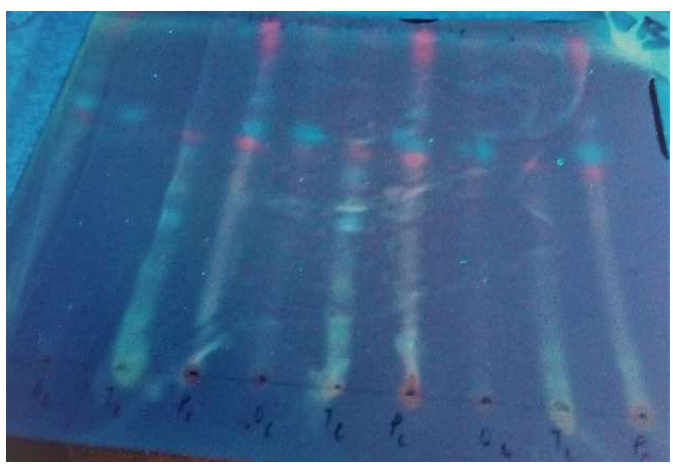

Figure 4f. $366 \mathrm{~nm}$ UV chromatogram of the ethyl acetate extracts after treatment with Libermann-Büchard

\subsection{Parameter calculations}

We segmented each spot of the images in Figure 4 above, then calculated mean intensity, standard deviation, and entropy using the algorithms coded in the Matlab 2016 version running under Microsoft Windows 10 Pro on a PC based on Intel i3. Figures $5 ; 6$ and 7 illustrate the segmentation of the different spots of the images used. We finally built a database with the values of the parameters of each task. The spots corresponding to families of molecules previously identified by the traditional technique of thin layer chromatography using developers.

- Average pixel intensity

Each spot on the plate is a grayscale digital image and an array of values. Each box in this table, which stores a value, is called a pixel. Noting $\mathrm{n}$ the number of lines and $\mathrm{p}$ the number of columns of the image, we manipulate an array of $\mathrm{n} \times \mathrm{p}$ pixels. Figure 5 , left, shows a visualization of a square array with $n=p=240$, which represents $240 \times 240=57600$ pixels. For an image, we can calculate the average intensity of pixels characterizing this image [10].

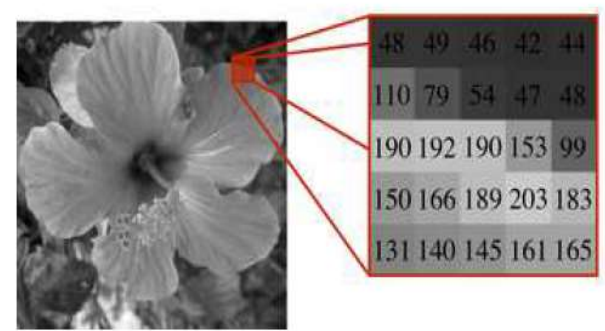

Figure 5. Under image (spot) size $5 \times 5$

The algorithm (a) below calculates the average pixel intensity of the spots. (See Figure 6)

$\mathrm{b}=$ imshow(im1);

im2=rgb2gray(im1);

$\mathrm{h}=$ imfreehand;

bw=createMask(h,b);

figure, imshow(bw)

$\mathrm{ht}=$ regionprops(bw,im2, 'MeanIntensity');

at $=$ ht.MeanIntensity 

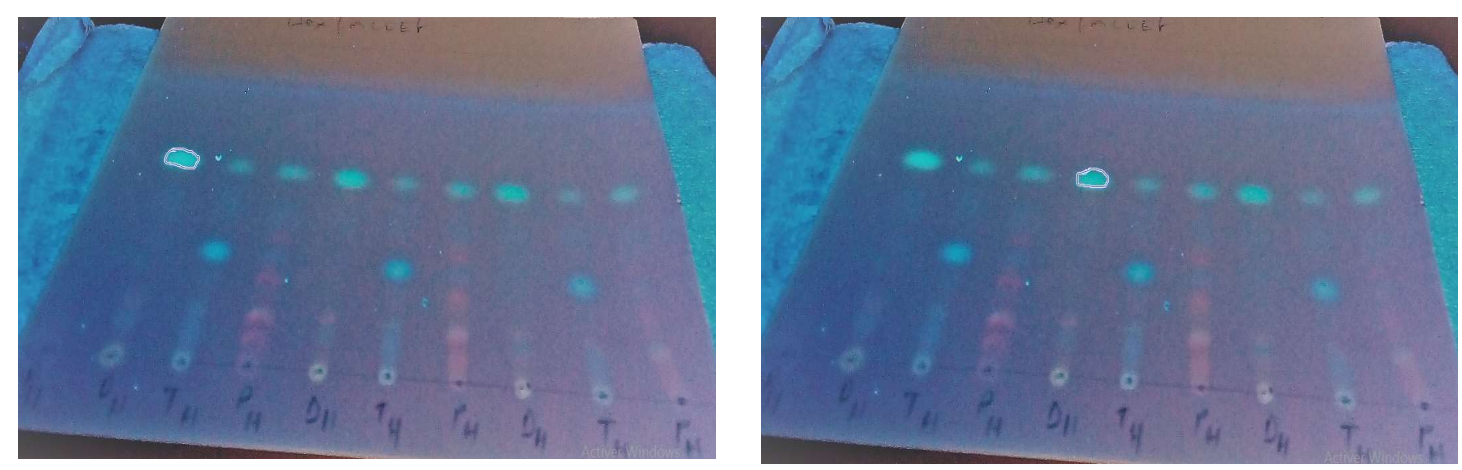

Figure 6. (a) Segmentation of spots under UV $366 \mathrm{~nm}$ corresponding to families of molecules
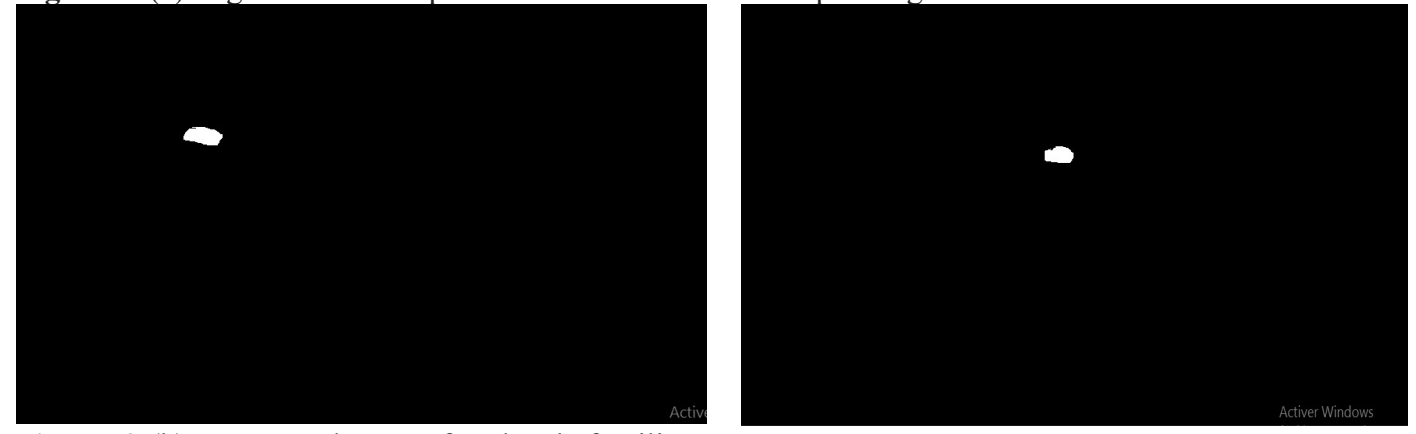

Figure 6. (b) Segmented spots of molecule families

- $\quad$ Standard deviation (Std):

The Std is defined by the equation (1) below.

$$
\left.S t d=\left(\frac{1}{n-1}\right) \sum_{i=1}^{n}\left(x_{i}-\bar{x}\right)^{2}\right)^{\frac{1}{2}}
$$

The algorithm (b) below allows to calculate the standard deviation of the spots. (See Figure 7)
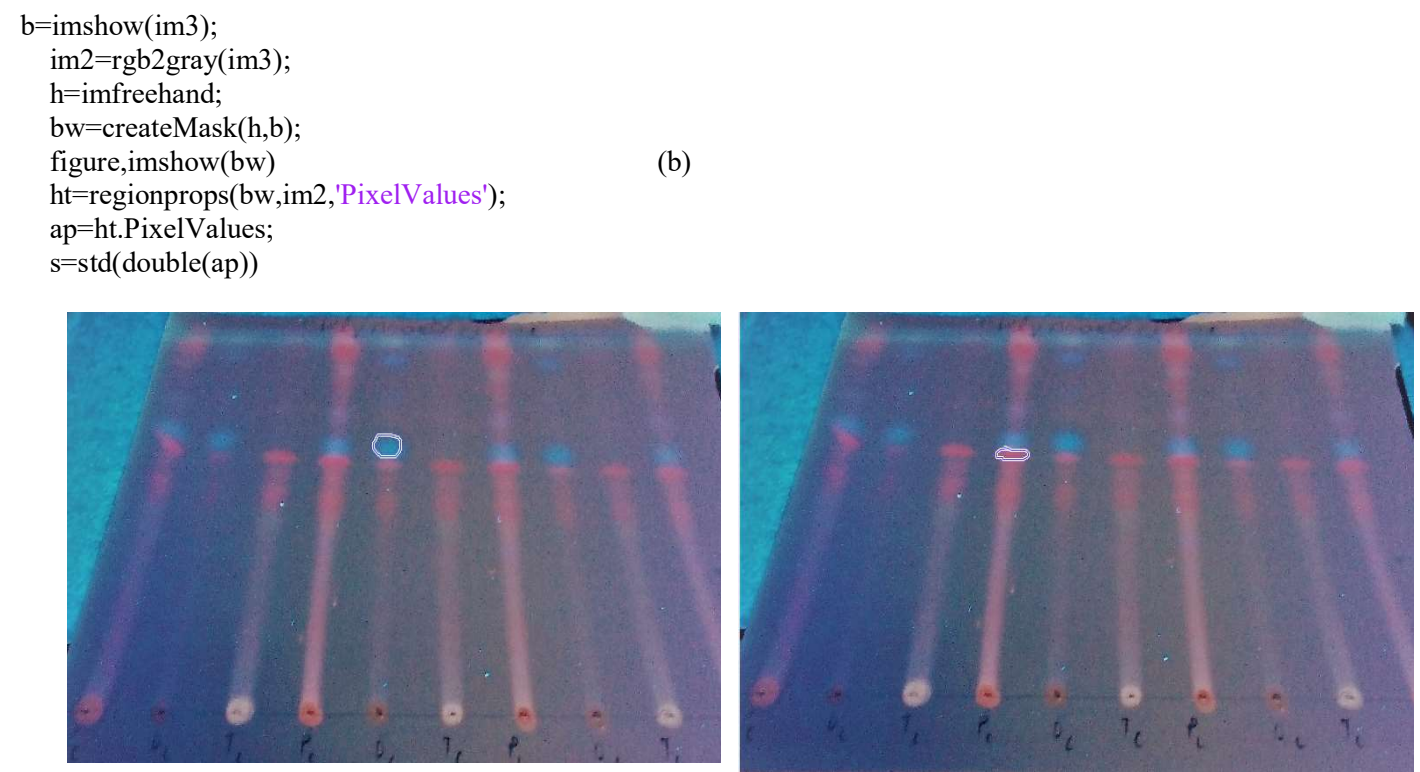

Figure 7. (a) Segmentation of spots under UV $366 \mathrm{~nm}$ corresponding to families of molecules 

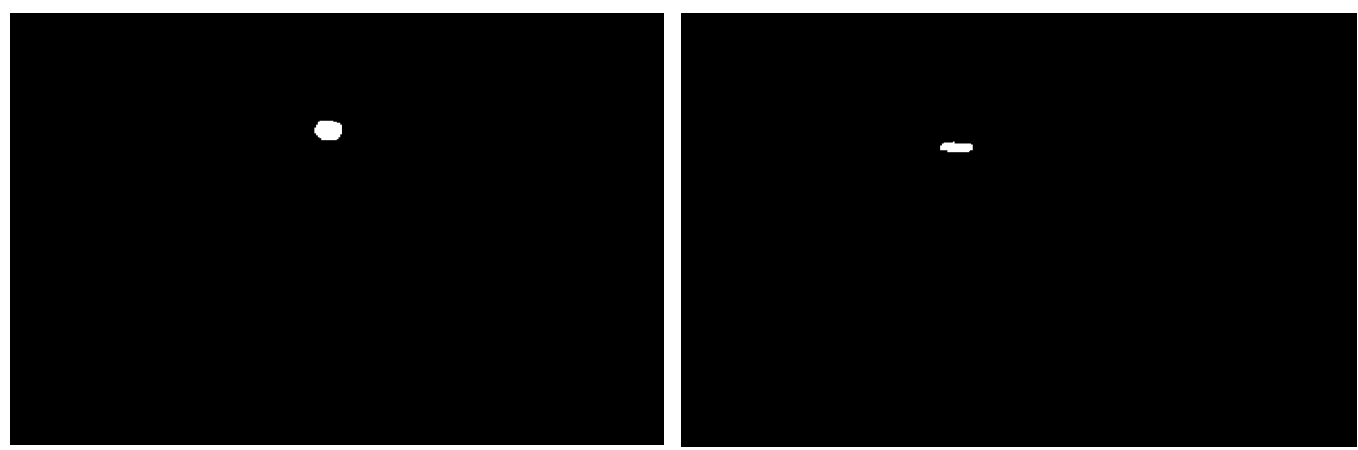

Figure.7 (b) Segmented spots of molecule families

- $\quad$ Entropy $(H)$ :

The entropy $H$ for a data source $X$ can be calculated as in (2).

$$
H(X)=-\sum_{i=1}^{n} P_{i} \log P_{i}
$$

The algorithm (c) below calculates the average pixel intensity of the spots. (see Figure 8 ) below

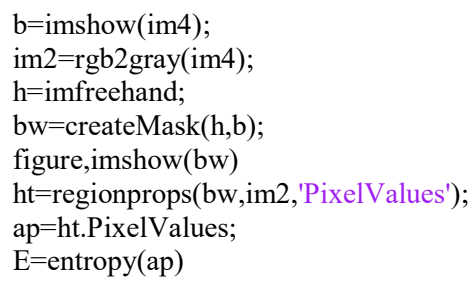

ht=regionprops(bw,im2,'PixelValues');

ap=ht.PixelValues;

$\mathrm{E}=\operatorname{entropy}(\mathrm{ap})$
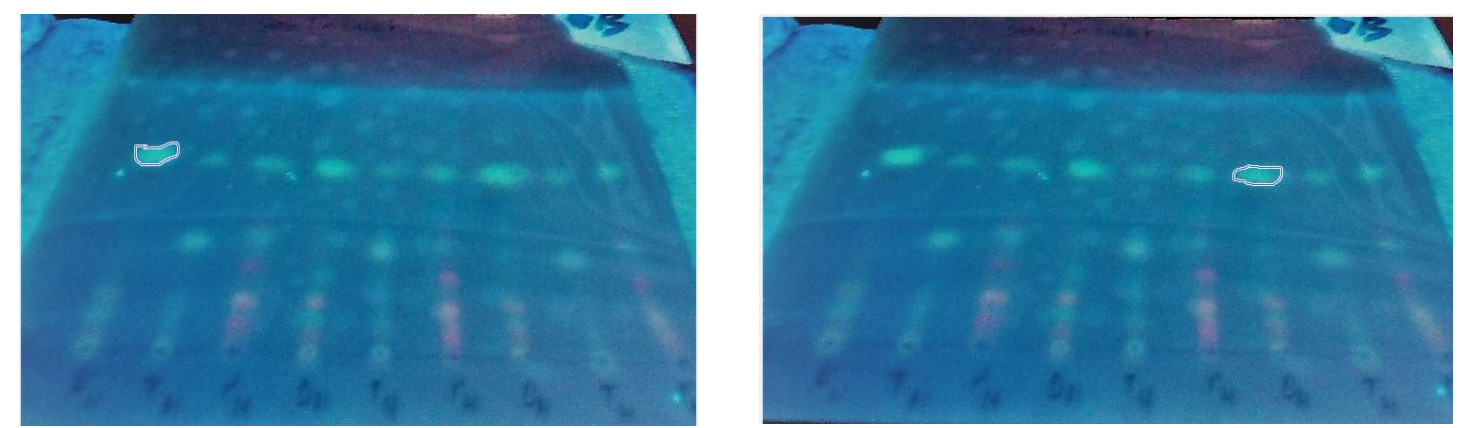

Figure 8. (a) Segmentation of spots under UV $366 \mathrm{~nm}$ corresponding to families of molecules
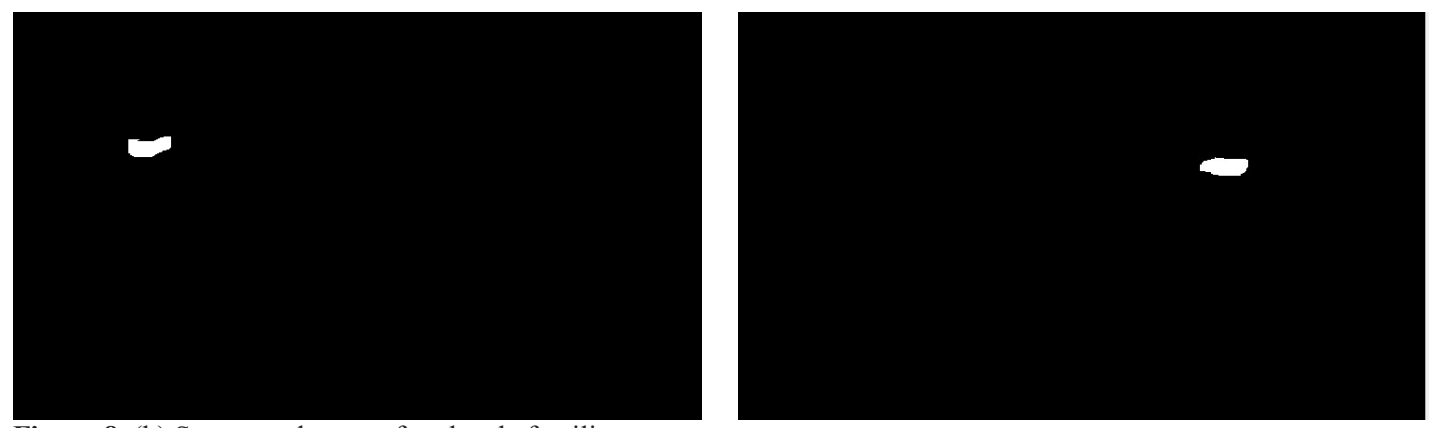

Figure 8. (b) Segmented spots of molecule families

\subsection{Parameter values}

For a spot on the plate, we identify the corresponding molecule family by the traditional method using the developers and for this task, parameters are calculated constituting a database and corresponding to this family of molecule. The parameter values for the families of molecules are given in Tables 1, 2 and 3 . 
Table 1: Parameter values of the families of molecules of the hexane fraction

\begin{tabular}{lclc}
\hline Molecules families & $\begin{array}{c}\text { MeanIntensity } \\
(\text { at })\end{array}$ & $\begin{array}{c}\text { Standard deviation } \\
(\text { Std })\end{array}$ & $\begin{array}{c}\text { Entropy } \\
(\mathbf{E})\end{array}$ \\
\hline coumarin & {$[81,700 ; 91,900]$} & {$[4,07 ; 4,60]$} & {$[2,04 ; 2,11]$} \\
Tannin & {$[11,880 ; 20,710]$} & {$[1,57 ; 1,70]$} & {$[4,11 ; 4,61]$} \\
Flavonoid & {$[201,78 ; 299,92]$} & {$[5,87 ; 5,90]$} & {$[3,11 ; 3,31]$} \\
Triterpenic genet & {$[186,04 ; 196,10]$} & {$[4,11 ; 4,20]$} & {$[3,50 ; 3,60]$} \\
hydroflavonol & {$[124,56 ; 131,60]$} & {$[4,50 ; 4,60]$} & {$[5,77 ; 5,87]$} \\
Terpene & {$[134,56 ; 144,60]$} & {$[6,54 ; 6,60]$} & {$[5,90 ; 5,99]$} \\
Steroid sterol & {$[201,88 ; 203,90]$} & {$[2,00 ; 2,11]$} & {$[4,46 ; 4,56]$} \\
anthocyanin & {$[193,11 ; 194,20]$} & {$[4,01 ; 4,41]$} & {$[4,31 ; 4,41]$} \\
polyphenol & {$[196,04 ; 198,10]$} & {$[4,91 ; 4,93]$} & {$[1,65 ; 4,71]$} \\
\hline
\end{tabular}

Table 2: Parameter values of the families of molecules of the chloroform fraction

\begin{tabular}{lclc}
\hline Molecules families & $\begin{array}{c}\text { MeanIntensity } \\
(\text { at })\end{array}$ & $\begin{array}{c}\text { Standard deviation } \\
(\text { Std })\end{array}$ & $\begin{array}{c}\text { Entropy } \\
(\mathbf{E ~})\end{array}$ \\
\hline coumarin & {$[81,700 ; 91,900]$} & {$[4,07 ; 4,60]$} & {$[2,04 ; 2,11]$} \\
Tannin & {$[11,880 ; 20,710]$} & {$[1,57 ; 1,70]$} & {$[4,11 ; 4,61]$} \\
Flavonoid & {$[201,78 ; 299,92]$} & {$[5,87 ; 5,90]$} & {$[3,11 ; 3,31]$} \\
Triterpenic genet & {$[186,04 ; 196,10]$} & {$[4,11 ; 4,20]$} & {$[3,50 ; 3,60]$} \\
hydroflavonol & {$[124,56 ; 131,60]$} & {$[4,50 ; 4,60]$} & {$[5,77 ; 5,87]$} \\
Terpene & {$[134,56 ; 144,60]$} & {$[6,54 ; 6,60]$} & {$[5,90 ; 5,99]$} \\
Steroid sterol & {$[201,88 ; 203,90]$} & {$[2,00 ; 2,11]$} & {$[4,46 ; 4,56]$} \\
anthocyanin & {$[193,11 ; 194,20]$} & {$[4,01 ; 4,41]$} & {$[4,31 ; 4,41]$} \\
polyphenol & {$[196,04 ; 198,10]$} & {$[4,91 ; 4,93]$} & {$[1,65 ; 4,71]$} \\
\hline
\end{tabular}

Table 3: Parameter values of the families of molecules of the acetate ethyl fraction

\begin{tabular}{lclc}
\hline Molecules families & $\begin{array}{c}\text { MeanIntensity } \\
(\text { at })\end{array}$ & $\begin{array}{c}\text { Standard deviation } \\
(\text { Std })\end{array}$ & $\begin{array}{c}\text { Entropy } \\
(\mathbf{E ~})\end{array}$ \\
\hline coumarin & {$[81,700 ; 91,900]$} & {$[4,07 ; 4,60]$} & {$[2,04 ; 2,11]$} \\
Tannin & {$[11,880 ; 20,710]$} & {$[1,57 ; 1,70]$} & {$[4,11 ; 4,61]$} \\
Flavonoid & {$[201,78 ; 299,92]$} & {$[5,87 ; 5,90]$} & {$[3,11 ; 3,31]$} \\
Triterpenic genet & {$[186,04 ; 196,10]$} & {$[4,11 ; 4,20]$} & {$[3,50 ; 3,60]$} \\
hydroflavonol & {$[124,56 ; 131,60]$} & {$[4,50 ; 4,60]$} & {$[5,77 ; 5,87]$} \\
Terpene & {$[134,56 ; 144,60]$} & {$[6,54 ; 6,60]$} & {$[5,90 ; 5,99]$} \\
Steroid sterol & {$[201,88 ; 203,90]$} & {$[2,00 ; 2,11]$} & {$[4,46 ; 4,56]$} \\
anthocyanin & {$[193,11 ; 194,20]$} & {$[4,01 ; 4,41]$} & {$[4,31 ; 4,41]$} \\
polyphenol & {$[196,04 ; 198,10]$} & {$[4,91 ; 4,93]$} & {$[1,65 ; 4,71]$} \\
\hline
\end{tabular}


For the different fractions, the parameters have the same values, this allows us to develop the identification program in Figure 9 below.

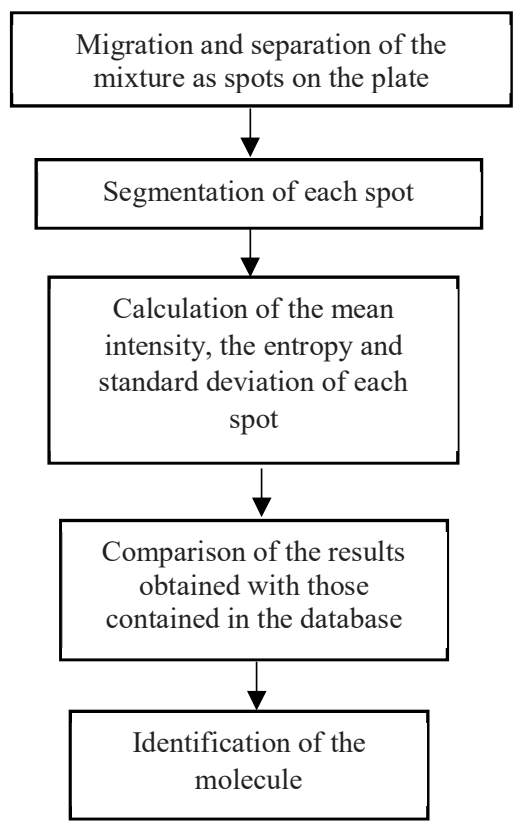

Figure 9. Program of identification of the molecule

We tested the presence of coumarins, tannins and terpenes in the hexane fraction of leaves of Blighia unijugata Bak (Sapindaceae).

The protocol of the thin layer chromatography being the same, we made the segmentation of the different spots on the chromatographic plate. We then calculated the different parameters of the image processing namely entropy, front ratio (Rf), standard deviation (std) and average pixel intensity (MeanIntensity). We obtained the results recorded in Table 4. These values correspond to molecules in the database.

Table 4: parameter values of the hexane fraction of blighia unijugata leaves

\begin{tabular}{ccc}
\hline $\begin{array}{c}\text { MeanIntensity } \\
\text { ( at ) }\end{array}$ & $\begin{array}{c}\text { Standard deviation } \\
\text { ( Std ) }\end{array}$ & $\begin{array}{c}\text { Entropy } \\
\text { ( E ) }\end{array}$ \\
\hline 89,01 & 4,11 & 2,08 \\
11,90 & 1,61 & 4,51 \\
135,04 & 6,58 & 5,96 \\
\hline
\end{tabular}

Since the values of the various parameters are defined, we have compared these results with the values in the database.

From Table 4 we can confirm the presence of coumarins, tannins, terpenes in the leaves of Blighia unijugata Bak (Sapindaceae) because the values obtained correspond to the values in the database. These molecules have already been identified by the traditional method with the same plants [11].

These results thus show the reliability of this identification technique and its simplicity in its implementation.

\section{Conclusion}

We exposed a new thin-film family identification technique based on image segmentation and calculation of segmentation parameters such as entropy, standard deviation, and average pixel intensity. Our method shows quite satisfactory results and have been confronted with the results of the technique traditionally made from the developers and the UV lamp $366 \mathrm{~nm}$ and $254 \mathrm{~nm}$. The results of this confrontation showed a similarity between the families of molecules obtained. This shows the reliability and robustness of our technique. 
In our next work, it will be a question of designing an electronic device coupled to a computer allowing to calculate automatically the entropy, the average intensity and the standard deviation of each spot in order to identify the family of molecule.

\section{References}

1. Muthu C., Aymar M., Raja N., and Ignacimuthu S. Medicinal plants used by Traditional healers in Kancheepuran District of Tamil Nadu, India. Journal of enthnobiological and ethnomedicine. 2006, 2 (43): 1186 / 1746-4269-2-43.

2. Kabran, Chemical and cytotoxic study of ten Ivory Coast plants, used in the traditional treatment of breast cancer; 2014

3. Adjanohoun E. \& Aké-Assi L. Contribution to the inventory of medicinal plants of Ivory Coast. Ministry of scientific research. National Floristic Center, Abidjan, Ivory Coast. 2008, 359 p

4. Bi F.H., Irié G.M., N'gaman K.C.C., Mohou C.H.B. Studies of some plants Therapeutics used in the treatment of high blood pressure and diabetes: two emerging pathologies in Côte d'Ivoire. 2008, Science \& Nature, 39-48 pp.

5. Adjanohoun E. \& Aké-Assi L. Contribution to the inventory of medicinal plants of Ivory Coast. Ministry of scientific research. National Floristic Center, Abidjan, Ivory Coast. 2008, 359 p.

6. Arbonier M. Trees, shrubs and lianas in the dry areas of West Africa. 2nd Edits CIRAD. 2002.

7. Obeng E.A. Blighia unijugata Baker. [Internet] Record from Protabase. Lemmens. 2010.

8. Zimudzi C. \& Cardon D., (2005). Morinda lucida Benth. [Internet] Record from PROTA4U. Jansen, P.C.M. \& Cardon, D. (Editors). PROTA (Plant Resources of Tropical Africa), Wageningen, Netherlands. $<$ Http://www.prota4u.org/search.asp >. Accessed June 13, 2013

9. Kabran, Chemical and cytotoxic study of ten plants from Côte d'Ivoire, used in the traditional treatment of breast cancer, 2014

10. Gabriel P. Digital processing of images. "Images of mathematics " .2011

11. Brou K.G., Mamyrbekova-Bekro J.A., Dogbo D.O., Gogbeu S.J., Bekro Y-A. On the Qualitative Phytochemical Composition of Crude Hydromethanolic extracts of theLeaves of 6 Varieties of Manihot Esculenta Crantz of Ivory Coast. European Journal of Scientific Research, 45 (2): 200-211. 2010. 